\title{
Towards Automated Smart Mobile Crowdsensing for Tinnitus Research
}

\author{
Muntazir Mehdi*, Denis Schwager*, Rüdiger Pryss ${ }^{\dagger}$, Winfried Schlee ${ }^{\ddagger}$, Manfred Reichert ${ }^{\dagger}$, Franz J. Hauck* \\ * Institute of Distributed Systems, Ulm University \\ $\dagger$ Institute of Databases and Information Systems, Ulm University \\ Email: \{muntazir.mehdi,denis-norbert.schwager,ruediger.pryss,manfred.reichert,franz.hauck\}@uni-ulm.de \\ $\ddagger$ Clinic and Policlinic for Psychiatry and Psychotherapy, Regensburg \\ Email: winfried.schlee@ tinnitusresearch.org
}

\begin{abstract}
Tinnitus is a disorder that is not entirely understood, and many of its correlations are still unknown. On the other hand, smartphones became ubiquitous. Their modern versions provide high computational capabilities, reasonable battery size, and a bunch of embedded high-quality sensors, combined with an accepted user interface and an application ecosystem. For tinnitus, as for many other health problems, there are a number of apps trying to help patients, therapists, and researchers to get insights into personal characteristics but also into scientific correlations as such. In this paper, we present the first approach to an app in this context, called TinnituSense that does automatic sensing of related characteristics and enables correlations to the current condition of the patient by a combined participatory sensing, e.g., a questionnaire. For tinnitus, there is a strong hypothesis that weather conditions have some influence. Our proof-of-concept implementation records weather-related sensor data and correlates them to the standard Tinnitus Handicap Inventory (THI) questionnaire. Thus, TinnituSense enables therapists and researchers to collect evidence for unknown facts, as this is the first opportunity to correlate weather to patient conditions on a larger scale. Our concept as such is limited neither to tinnitus nor to built-in sensors, e.g., in the tinnitus domain, we are experimenting with mobile EEG sensors. TinnituSense is faced with several challenges of which we already solved principle architecture, sensor management, and energy consumption.
\end{abstract}

Index Terms-mobile crowdsensing; tinnitus; automated mobile sensing; mobile health;

\section{INTRODUCTION}

Tinnitus is a common disorder, which is associated with the perception of a ringing sound or noise in the ears. The causative factors of tinnitus are still unknown, however, it usually is intertwined with an underlying condition in the ear. Little is also known about which external conditions, e.g., weather, and how they influence a patient's tinnitus experience.

Recently, smartphone apps and health appliances like heart and step meters got popular to help patients to maintain and mitigate their health problems. Smartphones are especially interesting as they nowadays come with high computational power, pose a good battery life time, and a set of built-in sensors that can easily be managed by apps. Even if there is a need for additional sensors, they can easily be connected (e.g., via BlueTooth). Further, smartphones come with an application ecosystem that can be extended by new apps, which are just software that can be programmed for a particular health problem. Finally, users are typically familiar to the touch- based interface, which can be used for customizations, but also to allow arbitrary input (e.g., questionnaires).

There are apps collecting patient data by asking for the patients' conditions (participatory sensing), which are relevant for therapists tracking these conditions during their patients' daily routine. In case of tinnitus, patients can fill-in apppresented questionnaires that can be analysed by researchers and therapists in order to gain new insights into tinnitus.

Other apps can continuously track patients' behaviour, like steps gone or heart-beat rates during the day. However, to the best of our knowledge, there is no app yet that continuously and automatically tracks patients' behaviour or other characteristics around patients (e.g., pressure, light, loudness, etc.) and correlates those to the patients' own condition (e.g., their annoyance or affection by tinnitus).

This paper presents TinnituSense, our novel concept and proof-of-concept implementation of an app tracking weather conditions and correlating them to the current patient condition by filing in a questionnaire. Researcher and therapists can use collected data for a per-patient analysis, but also for broader analyses of how tinnitus is influenced by weather conditions in general. Our concept is applicable to other domains, but focussed on tinnitus as it was developed as part of the European School for Interdisciplinary Tinnitus Research $(\text { ESIT })^{1}$.

We organised this paper as follows: Section II reports on related work. In Section III, we will give some background and motivation for our work. While Section IV describes our approach, its implementation, and our solution of challenges. Then, in Section V, we present first results and their impact. Finally, Section VI concludes our work and provides some future considerations.

\section{RELATED WORK}

With respect to employing mobile crowdsensing in the healthcare domain, various solutions have been proposed in literature. For instance, in case of individual-centric applications, Györbíró et al. propose an activity and posture detection mechanism [6]. A more detailed use-case-based

\footnotetext{
${ }^{1}$ funded by the EU Horizon 2020 program as Marie Skłodowska-Curie grant, agreement number 722046
} 
solution, specifically targeted towards activity detection of patients suffering from Parkinson's disease is reported in [2]. Additionally, the authors in [17] and [14] report image-based and video-imaging-based solutions for diet and heart-rate monitoring. An attempt to use social-media data to monitor emotional health of patients is presented in [10].

Similarly, in case of environment-centric applications, [4] presents an approach that enables use of specialised auxiliary sensors to achieve participatory sensing for active monitoring of air quality and pollution. Authors of [8] present a locationand machine-learning-based solution towards observance of disease and disaster outbreak. Both of these approaches rely highly on participatory sensing coupled with minor automated sensing, carried out by auxiliary or specialised sensors.

Within the scope of tinnitus research, a plethora of scientific literature report different applications of mobile crowdsensing, ranging from data collection to mitigating tinnitus symptoms and supporting clinicians. For instance, the smartphone app TrackYourTinnitus, based on participatory sensing, systematically records fluctuations of tinnitus symptoms from patients. With these records a connection between tinnitus and daily routine or activities [15] can be established. Unlike TrackYourTinnitus, our TinnituSense approach supports automated sensing of weather-related data in addition to feedback (in form of THI surveys) from the patients.

NoiseTube [11] is another pertinent app that acquires geolocalised noise levels using the embedded microphone of the smartphone. This data is therefore examined by scientists to identify relationship between behavioural and psychological problems associated with noise pollution. Similarly, Sound Meter uses the microphone of mobile devices to measure the environment loudness and gives a reference sound to compare the loudness with [13]. Unlike both apps using only microphone and GPS to measure the environment, TinnituSense uses several additional sensors, such as the photometer, barometer, thermometer, hygrometer, accelerometer, and gyroscope to get additional information about the environmental condition.

\section{BACKGROUND AND MOTIVATION}

\section{A. Tinnitus}

As mentioned, tinnitus is a disorder associated with the perception of irritating sound or noise. In addition to general health complications, tinnitus might be also responsible for provoking other psychological disorders (e.g., stress, anxiety, depression, or obsessive-compulsive disorder) and may affect the common as well as social lifestyles. Furthermore, tinnitus has also been described to may have correlations with migraine and vertigo. The perception of tinnitus is driven by the filtering work of subconscious areas in the brain-stem influenced by the limbic system, and increases in episodes of interior or exterior stress.

Changes in the atmospheric surrounding may have direct or indirect effects on the annoyance caused by tinnitus. Some scientific literature has reported an alleviation in the tinnitus condition of patients with Meniere's Disease, which can be related to the surrounding atmospheric and environmental conditions [19]. Among others, factors like a decrease in the atmospheric pressure, a weather change (specifically rainy and cold weather), intensity of light, the current sound environment, or (sudden) change in altitude are some of the most pertinent ones. Importantly, weather is no causative factor for tinnitus, but may influence the perception of the sounds. Very often patients report that they hear intermittent secondary tones to their common tinnitus sound or describe their common tone changing from compensated to annoying expressed by terms like hammering, beating, fizzling.

Similarly, air pressure is considered to be responsible for episodes of migraine in many patients [9]. In rare cases, patients describe a change in tinnitus perception corresponding to their migraine in major air pressure changes. On the other hand, the orientation, movement speed, light intensity, and direction of movement of the patient are some of the less common, yet still significant set of factors that may also induce a spike in tinnitus symptoms.

Tinnitus patients often report that the perception of their tinnitus varies. The tinnitus loudness, as well as the tinnitusrelated distress, can change from one moment to the other. This moment-to-moment variability can be measured with Ecological Momentary Assessment methods [18]. Even though some influencing factors for this variability has been identified [16], a large amount of the variability cannot be explained. With this project, we also want to investigate the influence of weather conditions on the individual perception of tinnitus.

\section{B. TinnituSense Approach}

We designed TinnituSense as a concept for mobile sensing and crowdsensing to enable advanced tinnitus research. Fig. 1 shows the actors in the TinnituSense use cases: patients, researchers, therapists, and a backend storing collected data. In [12], we presented a reference architecture that covers all aspects of a TinnituSense-like application, e.g., mobile sensor management, local and backend services, data management, analytics, etc. As mobile sensing can be applied to monitor the aforementioned atmospheric factors (most, if not all) that provoke or influence tinnitus, this paper presents a proof-ofconcept app for this purpose. Our contribution compared to previous work is the correlation of weather conditions to the condition of the patient. This is done by not only automatically collecting sensor data, but to also connecting them to the patient's current experience by using the Tinnitus Handicap Inventory (THI) ${ }^{2}$, a standardized questionnaire.

In principle, TinnituSense could be extended to further aspects: (i) The app could identify (drastic) changes in the circumstances (motion, environment, and position) of a patient who is prompted to fill-in a questionnaire about the severity of their current situation. These circumstances could be preconfigured by researchers or therapists. (ii) In detected circumstances the app could, configured by therapists, help to mitigate problems, e.g., by acoustic therapy (calming sounds, music therapy, listening pink or brown noise), intake of

\footnotetext{
${ }^{2}$ http://www.tinnituslab.com/Questionnaire_THI_ENGLISH.pdf
} 


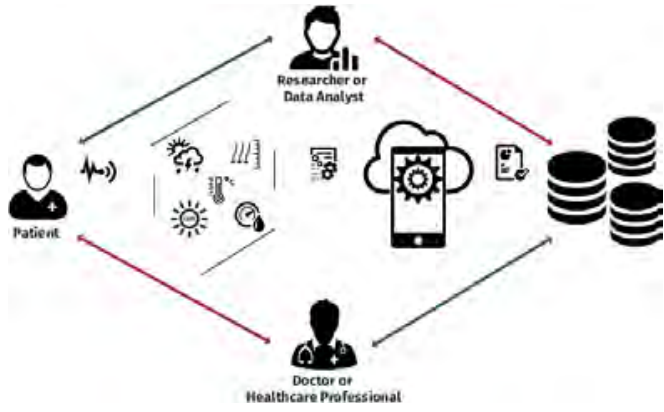

Fig. 1: TinnituSense Principle Actors

supplements, or personalised sound frequency treatments. (iii) Feedback to the patients about correlations may keep the patient aware of specific triggers and what may cause their tinnitus symptoms.

However, applying smart mobile crowdsensing in realworld scenarios brings forward a couple of technical challenges. Even though modern smart phones provide sophisticated hardware, their manufacturers intended to make these smartphones general purpose appliances. Herein, for sensing in the healthcare domain, it is pertinent to acquire accurate data, and since the sensors embedded in smartphones are not dedicated, the problem of inaccurate sensor data arises [7], in addition to the reliance on the sensed data [3]. In order to achieve more accurate sensed data, continuous sampling of the sensor data is required, which results in battery consumption problems [21]. Moreover, as the smart phones are resource constrained, employing smart mobile crowdsensing introduces scarcity of hardware for general user experience [1]. And in order to gain full potential of the mobile crowdsensing application, it is pertinent that the sensed data is transmitted to some cloud or backend, where it is further processed. This, in turn, introduces the data transmission challenges [5], as well as its associated energy consumption problem [20].

\section{IMPLEMENTATION}

The current implementation of the TinnituSense App has been carried out for Android platform using Google's dedicated Integrated Development Environment (IDE)-Android Studio $^{3}$. The current version of the app uses a combination of Android's standard libraries as well as OnsenUI ${ }^{4}$ to provide rich user experience. Currently, TinnituSense supports the following sensors:

- Photometer (light sensor): Measures the luminance in the unit Lux (lux)

- Barometer (air pressure sensor): Measures the ambient air pressure in the unit Hectopascal (hPa)

- Thermometer (temperature sensor): Measures the ambient air temperature in the unit Degree Celsius $\left({ }^{\circ} \mathrm{C}\right)$

- Hygrometer (humidity sensor): Measures the ambient relative humidity in percent $(\%)$

\footnotetext{
${ }^{3}$ https://developer.android.com/studio/

${ }^{4}$ https://onsen.io/
}

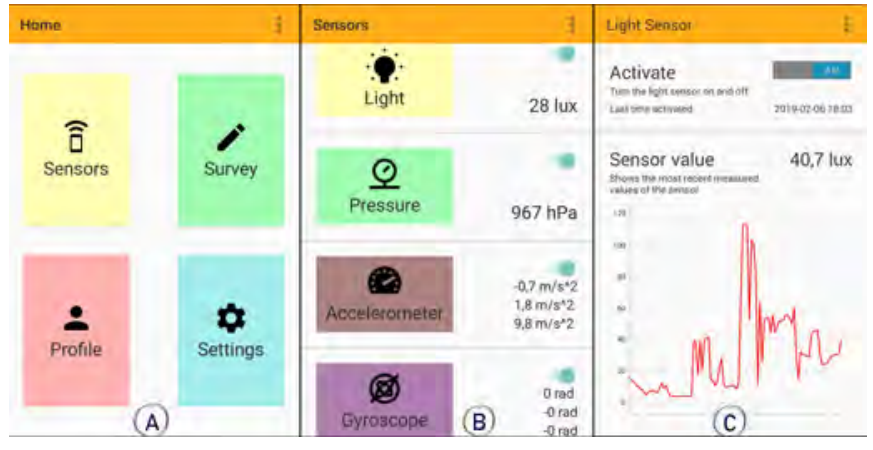

Fig. 2: A. Home Screen - B. Sensors Page - C. Sensor Detail

- Accelerometer (acceleration sensor): Measures the velocity along the $\mathrm{x}-, \mathrm{y}-$ and $\mathrm{z}$-axis (including gravity) in the unit Meters per Second Squared $\left(\frac{m}{s^{2}}\right)$

- Gyroscope (angular rate sensor): Measures the rate of rotation around the $\mathrm{x}-, \mathrm{y}-$ and $\mathrm{z}$-axis in the unit Radiant per Second $\left(\frac{\mathrm{rad}}{\mathrm{s}}\right)$

On first time invoking the app, it initially probes the embedded sensors and identifies the supported sensors by the device. In case, if any of above mentioned sensors is not supported, the app displays the 'Not Supported' message to the user. These settings are then stored locally to avoid future probes. Furthermore, the user gets a 'Home' Screen (cf. in Fig. 2(A)) with four navigational buttons. Each button has textual descriptions as well as pictorial representations of the underlying function. Additionally, an easy-access navigational sub-menu button on the upper right corner is accessible throughout every screen in the app. The sub-menu enables quick access to aforementioned four navigational buttons as well as the home screen.

The Survey button (cf. Fig. 2(A)) invokes the Tinnitus Handicap Inventory (THI) questionnaire within the app. If a user completes and submits answers to the survey, the app scores the answers based on the THI scale. This score, answers to the survey questionnaire, timestamp of survey response are then stored primarily on the device, and once a data connection has been established based on user's preference (WiFi or Mobile), this data is then transmitted to a backend. In addition to wilfully filling out the survey at any time, the user is also periodically prompted via app notifications.

The Settings enable app customizations, like enabling day or night mode as a theme. Enabling or disabling app notifications, changing the frequency of THI survey prompts (e.g., weekly, monthly etc.), and data transmission options, for instance via WiFi connection or mobile data connection.

Since TinnituSense promotes crowdsensing, it is pertinent that individual profiles are generated to uniquely identify patients. The Profile section of the app thus acquires userspecific data. This data is sub-divided into two major parts, primarily, the user provides basic (so called 'non-personal') information about themselves like age and gender. We ensure that we do not ask any personal questions like name, and date of birth. Secondarily, the app asks the user about tinnitusrelated questions, for instance, first encounter with tinnitus, 
type and severity of tinnitus, therapy, and medication related questions. In addition to having a user-specific profile, this information is also used to generate a unique profile identifier.

As sensors are important participants of the app, information specific to their current activity is hence deemed critical. This is therefore, tapping the Sensors button (cf. Fig. 2 (A)), will lead the user to list of sensors supported by the device, screenshot of this is depicted in Fig. 2 (B). Herein, each supported device sensor is shown using a text and a pictorial representation (icon), furthermore, the user can also see the current sensor value, as well as has the option to turn a device sensor ON or OFF. Since, we do not automatically manage the activation and deactivation of the sensors within the app, we leave it to user's judgement to enable and disable a certain sensor based on its battery consumption and significance.

We also identified that the user would be interested in visualising the sensor values to gain an insight into his current surrounding environment. In terms of usability, provision of an observable action by the sensors would gain user's trust in the application. Therefore, tapping on any sensor in Fig. 2 (B), will lead the user to sensor-specific page. An example of such a page for 'Light Sensor' is shown in Fig. 2 (C). The sensor specific page shows the live sensor value in addition to a line chart with the history of the most recent measurements. The chart is updated with every new measurement and the ranges on the $\mathrm{x}$ - and $\mathrm{y}$-axes are adjusted dynamically. We believe that the line chart would be sufficient for a user to properly interpret the sensor values, enabling him to identify peak and low values and compare with current tinnitus sensation. The user also has the option to activate or deactivate a sensor within the sensor specific page. And a timestamp showing the time the sensor was activated is also shown.

As mentioned before, the activation and deactivation of a sensor is dependent on user's judgement. We employ this scheme to provide users with sufficient freedom to control critical aspects of the app. However, we ensure to support user's judgement by provision of necessary values regarding the battery consumption of individual sensor. The different variations of available information regarding the battery consumption of individual sensor are shown in Fig. 3. Where Fig. 3 (A) provides information on current battery usage and a bar-chart showing the battery consumption of the sensor for past 5 activation periods, both values are given in $m A h$. Every new bar in the chart is rendered at the moment the user turns the corresponding sensor off. Fig. 3 (B), shows a pie-chart comparing the current battery consumption of the sensor with overall capacity of the device battery, herein, the red line signifies the power consumed by 'Light Sensor' since its last activation, while the grey colour signifies the battery capacity (dark grey for available battery, and light grey for used battery). And in Fig. 3 (C), we show a pie-chart depicting the current battery usage of the sensor in relation to other active sensors of the device as well as the remaining and used battery of the device in $m A h$. The legend at the bottom describes the colour codes associated with their respective device sensors. Both of these pie-charts are updated at the

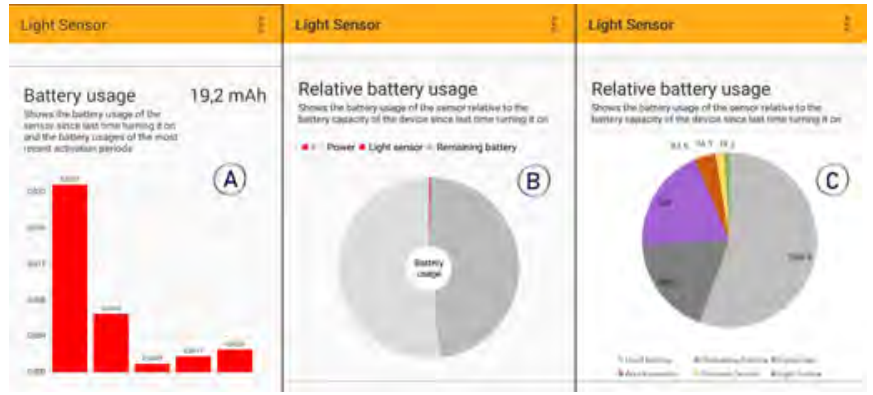

Fig. 3: Battery Usage :- A. Last 5 activations B. In relation to device battery C. In relation to other sensors and device battery

interval of 30 seconds.

We argue that the battery consumptions of individual sensors, in relation to the device battery, and other sensors will provide the user with necessary information to support the decision of turning a sensor ON or OFF. For instance, from Fig. 3 (C), the user can easily identify that the gyroscope is consuming large amount of battery, and if the user deems that the gyroscope is not significant in relation to his tinnitus, he can decide to turn the gyroscope sensor OFF. Similarly, this will enable the user to experiment with multiple sensors while conserving the battery to identify sensors which are critical in terms of his tinnitus sensation. For example, for a user whose tinnitus symptoms worsen due to changes in altitude, the barometer sensor will play a significant role.

\section{RESUlTS AND DISCUSSIONS}

To check the applicability, usability, and practicality of the proposed approach and the app, we performed both quantitative and qualitative tests. For quantitative tests, we used two standard smartphones in default configuration, Google Nexus 4 with Android version 6.0 and Samsung Galaxy with Android version 4.4.

As, it is possible for a user to turn $O N$ and $O F F$ the device sensors using the app, an indication of individual sensor's battery (power) consumption can be helpful in managing the over-all battery of the device. To better understand and to get an indication of how much battery capacity is consumed by the individual sensors of the two devices, four different tests were carried out over a period of 12 hours. An average battery consumption of individual sensor per device is shown in Table I. It is evident that the Barometer of both devices uses the least energy, while the Gyroscope consumes the most. In all test runs, the battery consumption is determined by activation time (in ms) multiplied by strength of electric current of the corresponding sensor (in $\mathrm{mAh}$ ).

\begin{tabular}{ll|l|l|}
\multirow{2}{*}{} & & \multicolumn{2}{|l|}{ Average battery usage in $\boldsymbol{m A h}$ per $\mathbf{1 2 h}$} \\
\cline { 3 - 4 } & Sensor & Google Nexus 4 & Samsung Galaxy \\
1. & Photometer & 2.11 & 8.95 \\
2. & Barometer & 0.07 & 0.26 \\
3. & Thermometer & unsupported & 7.96 \\
4. & Hygrometer & unsupported & unsupported \\
5. & Accelerometer & 6.1 & 1.68 \\
6. & Gyroscope & 43.28 & 73.51
\end{tabular}

TABLE I: Device - Sensor: Battery consumption 


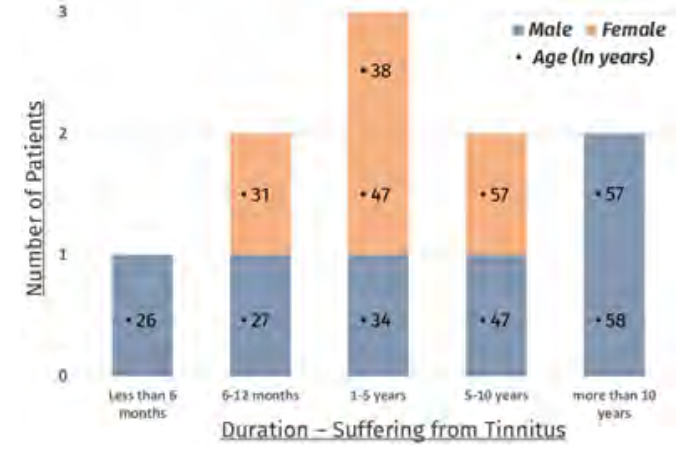

Fig. 4: Patient Demographics and Tinnitus Durations

Herein, since it could be argued that the sensor's battery consumption is subjective to the amount of its usage (for instance, the data it collects and forwards to the app), therefore, we ensured that the battery consumption of each sensor is computed while the sensor is completely activated in an ideal environment, and also probing it periodically for data. For example, for light sensor (photometer), Android system only activates and shares the sensor data when it detects changes in light intensity. Therefore, for testing purposes, we ensured that sensor was exposed to different light intensities and in addition to data being pushed from the light sensor, we implemented a pull mechanism that periodically requested data from sensor. During these tests, another keen observation was that the two devices generated varying measurements for some sensors in same circumstances. We believe that this could be resulting from the sensors being differently calibrated on both devices.

Further, we wanted to highlight the impact and need of a mobile crowdsensing app, like TinnituSense. We were interested in the opinion of the patients in terms of using such an app, and wanted to identify the reality between weatherrelated changes and their impact on tinnitus symptoms from the perspective of a patient. Finally, we liked to see the significance of medication or therapy-related solutions. Therefore, we conducted qualitative tests in form of 10 personal interviews with patients suffering from tinnitus at ENT Clinic of the German Army Hospital of Ulm.

The demographics of these patients, in terms of age, gender, and the duration of tinnitus are given in chart depicted in Fig. 4. The $x$-axis represents the duration or how long a patient has been suffering from tinnitus, while the $y$-axis gives the number of patients. Gender is separated by different colour codes, while the age (in years) is shown within individual blocks. For instance, there are 2 patients, 1 male aged 27 and 1 female aged 31, who have been suffering from tinnitus for 612 months. Within these diverse set of interviewees, 8 of them reported their tinnitus being caused by stress, while 3 also mentioned sound exposure. There was 1 infection-related case, and 1 listed their tinnitus caused by unknown reasons. On asking about their tinnitus severity on the scale of light, mild, moderate, severe, and catastrophic, 2 patients reported mild, 5 moderate, and 3 patients reported severe tinnitus burden. $80 \%$ of the patients reported accompanying dizziness, while $50 \%$ mentioned hearing loss caused by tinnitus.
None of the patients had ever taken a THI questionnaire. Among those patients, 50\% reported that their tinnitus symptoms worsen due to changes in weather ( 2 reported summer being critical, 2 reported winter, and 1 patient reported drastic changes to be elevating factor in tinnitus symptoms). Furthermore, on asking about their general (job, shopping, cooking) and social (party, gatherings, dinners) activities, 50\% of the patient responded that they reduce their general activity, while $70 \%$ reported avoiding social activities. Out of 10 , a total of 6 people reported use of medication to control tinnitus, among these, only 5 patients described medicines to be useful. Similarly, on asking if therapeutic solutions helped, all patients responded positive. Additionally, we asked patients if they used smartphones or any specific apps to help control their tinnitus, every patient responded positive to having a smartphone, while all of them responded negative to use of any application. Based on this, we asked if they would be interested in using such an app (while explaining the TinnituSense app), 9 out of 10 patients agreed. And all these 9 out of 10 patients agreed to sharing their tinnitus related data for research purposes.

\section{FUtURE WORK AND CONCLUSION}

The current version of the TinnituSense app utilizes sensors which are embedded in most commonplace smartphones, and attempts at acquiring environmental data and monitors patient activity using the aforementioned sensors. This can help in understanding correlations between environmental or weather related changes with tinnitus symptoms. However, within the context of tinnitus research, there is still room to implement auxiliary sensors. For instance, mobile Electroencephalography (EEG) systems coupled to smartphones to acquire electrical activity of the brain, and enabling provision of realtime neurofeedback to the patients suffering from tinnitus. Additionally, the data acquired from mobile EEG systems can also be helpful for researchers and psychologists to better understand tinnitus and its associated brain activity. To establish a correlation between weather and tinnitus, the current version of the app implements the THI questionnaire. Herein, we believe that the app could benefit from adding tinnitus loudness and tinnitus distress related questions from TrackYourTinnitus app, as well as comparing the data from both apps. Currently, the app generated data (sensors, questionnaires, profile, settings, etc.) are stored locally on the device, and are transmitted to the backend once a data connection is established. However, we are presently working on a sophisticated Sensor Data Storage and Transmission technique by means of employing 1) $A d$ hoc Storage and 2) Distributed Processing. Where, Ad-hoc Storage will ensure storage of acquired sensor data on the device in compressed and optimal format in absence of data connection. While, Distributed Processing will find a balanced distribution of raw sensor data to be processed by the device and the backend. This, in turn, will enable better device battery life conservation, reduction of data transmission overheads and save processing resources of the device. 
Furthermore, even though the current implementation of TinnituSense attempts at conserving energy, there is still significant room for improvements. For instance, as of now, the app relies on Android's internal power management and depends on user's own will to manage individual sensor's battery consumption by turning it on/off manually. Herein, an automated sensor management technique, which identifies non-significant use of sensor and turns it off, as well as activation of high battery consuming sensors based on a criteria can further benefit the battery conservation. Moreover, to improve manual intervention of user to conserve battery by turning a sensor off (cf. Table I showing high battery consumption by the gyroscope), a notification scheme that reminds the user that a sensor with high battery consumption has been running for a longer period of time could be used, so that the user can decide whether or not it would be better to turn off this sensor to save energy. As we highlighted previously, that the embedded sensors of smartphones are general purpose and therefore prone to data accuracy problems, the app could benefit from employing techniques that could drastically improve the data accuracy issues. For instance, achieving sensor fusion of temperature sensor by comparing it with third-party mobile apps (for example, Google Weather), could help in identifying error delta and thus re-calibrating the sensor can improve accuracy.

In this paper, we highlight the significance of mobile crowdsensing in the healthcare domain, specifically in tinnitus research. We reported the lack of automated sensing apps for tinnitus research and identify the technological gap that limits scientific studies to correlate environmental and weather changes to tinnitus symptoms. Therefore, we presented an approach towards automated sensing of environmental conditions and patient monitoring, backed by the use-case of tinnitus research. The approach is sustained by implementation of a smartphone app, a user-interface, and implementation of a usecase specific questionnaire. We believe that the technological steps undertaken in this paper will help in carrying out large scale scientific studies to quantify data correlating tinnitus symptoms with weather related and environmental changes. Results of a small survey on patients gives hope that patients are interested in using such an app. Thus, we believe that enabling the technology is one of the significant contributions of this paper. Furthermore, we argue that a sophisticated mobile crowdsensing app designed for monitoring patient surroundings and profiling patients for personalised therapy may foster controlling and mitigating of tinnitus symptoms, as well as promote community or participatory sensing.

\section{REFERENCES}

[1] Saeid Abolfazli, Zohreh Sanaei, and Abdullah Gani. Mobile cloud computing: A review on smartphone augmentation approaches. arXiv preprint arXiv:1205.0451, 2012.

[2] Mark V Albert, Santiago Toledo, Mark Shapiro, and Konrad Koerding. Using mobile phones for activity recognition in parkinson's patients. Frontiers in Neurology, 3:158, 2012.

[3] Jeffrey R Blum, Daniel G Greencorn, and Jeremy R Cooperstock. Smartphone sensor reliability for augmented reality applications. In
Int. Conf. on Mobile and Ubiq. Sys.: Comp., Netw., and Services, pages 127-138. Springer, 2012.

[4] Prabal Dutta, Paul M Aoki, Neil Kumar, Alan Mainwaring, Chris Myers, Wesley Willett, and Allison Woodruff. Common sense: participatory urban sensing using a network of handheld air quality monitors. In Proc. of the 7th ACM Conf. on Emb. Netw. Sensor Sys. (SenSys), pages 349-350. ACM, 2009.

[5] Raghu K Ganti, Fan Ye, and Hui Lei. Mobile crowdsensing: current state and future challenges. IEEE Comm. Mag., 49(11), 2011.

[6] Norbert Györbíró, Ákos Fábián, and Gergely Hományi. An activity recognition system for mobile phones. Mobile Netw. and App., 14(1):8291, 2009.

[7] Mohammad Ashfak Habib, Mas S Mohktar, Shahrul Bahyah Kamaruzzaman, Kheng Seang Lim, Tan Maw Pin, and Fatimah Ibrahim. Smartphone-based solutions for fall detection and prevention: challenges and open issues. Sensors, 14(4):7181-7208, 2014.

[8] Peter Haddawy, Lutz Frommberger, Tomi Kauppinen, Giorgio De Felice, Prae Charkratpahu, Sirawaratt Saengpao, and Phanumas Kanchanakitsakul. Situation awareness in crowdsensing for disease surveillance in crisis situations. In Proc. of the 7th Int. Conf. on Inform. and Comm. Techn. and Dev. (ICOICT), page 38. ACM, 2015.

[9] Kazuhito Kimoto, Saiko Aiba, Ryotaro Takashima, Keisuke Suzuki, Hidehiro Takekawa, Yuka Watanabe, Muneto Tatsumoto, and Koichi Hirata. Influence of barometric pressure in patients with migraine headache. Internal Medicine, 50(18):1923-1928, 2011.

[10] Mark E Larsen, Tjeerd W Boonstra, Philip J Batterham, Bridianne O'Dea, Cecile Paris, and Helen Christensen. We feel: mapping emotion on twitter. IEEE J. Biomed. and Health Inf., 19(4):1246-1252, 2015.

[11] Nicolas Maisonneuve, Matthias Stevens, Maria E Niessen, and Luc Steels. Noisetube: Measuring and mapping noise pollution with mobile phones. In Inform. Techn. in Environm. Eng., pages 215-228. Springer, 2009.

[12] Muntazir Mehdi, Guido Mühlmeier, Kushal Agrawal, Rüdiger Pryss, Manfred Reichert, and Franz J.Hauck. Referenceable mobile crowdsensing architecture: A healthcare use case. In Proc. of the 1st Int. Worksh. on Serv. for Mobile Data Coll. (MoDaC), volume 134, pages 445-451. Procedia Computer Science, 2018.

[13] Enda Murphy and Eoin A King. Testing the accuracy of smartphones and sound level meter applications for measuring environmental noise. Applied Acoustics, 106:16-22, 2016.

[14] Ming-Zher Poh, Daniel J McDuff, and Rosalind W Picard. Non-contact, automated cardiac pulse measurements using video imaging and blind source separation. Optics Express, 18(10):10762-10774, 2010.

[15] Thomas Probst, Rüdiger Pryss, Berthold Langguth, and Winfried Schlee. Emotional states as mediators between tinnitus loudness and tinnitus distress in daily life: Results from the "TrackYourTinnitus" application. Scientific reports, 6:20382, 2016.

[16] Thomas Probst, Rüdiger C Pryss, Berthold Langguth, Josef P Rauschecker, Johannes Schobel, Manfred Reichert, Myra Spiliopoulou, Winfried Schlee, and Johannes Zimmermann. Does tinnitus depend on time-of-day? an ecological momentary assessment study with the trackyourtinnitus application. Frontiers in aging neuroscience, 9:253, 2017.

[17] Sasank Reddy, Andrew Parker, Josh Hyman, Jeff Burke, Deborah Estrin, and Mark Hansen. Image browsing, processing, and clustering for participatory sensing: lessons from a dietsense prototype. In Proc. of the 4th Worksh. on Emb. Netw. Sensors (EmNetS), pages 13-17. ACM, 2007.

[18] Winfried Schlee, Rüdiger C Pryss, Thomas Probst, Johannes Schobel, Alexander Bachmeier, Manfred Reichert, and Berthold Langguth. Measuring the moment-to-moment variability of tinnitus: the trackyourtinnitus smart phone app. Frontiers in aging neuroscience, 8:294, 2016.

[19] Wiebke Schmidt, Natalie Sarran, Christophe an Ronan, George Barrett, David J Whinney, Lora E Fleming, Nicholas J Osborne, and Jessica Tyrrell. The weather and meniere's disease: a longitudinal analysis in the uk. Otology \& Neurotology, 38(2):225, 2017.

[20] Haoyi Xiong, Daqing Zhang, Leye Wang, and Hakima Chaouchi. Emc 3: Energy-efficient data transfer in mobile crowdsensing under full coverage constraint. IEEE Trans. on Mobile Comp., 14(7):1355-1368, 2015.

[21] Zhenyun Zhuang, Kyu-Han Kim, and Jatinder Pal Singh. Improving energy efficiency of location sensing on smartphones. In Proc. of the 8th Int. Conf. on Mobile Sys., App., and Services (MobiSys), pages 315330. ACM, 2010 